\title{
DO WE NEED TO WEIGHT ITEM SATISFACTION BY ITEM IMPORTANCE? A PERSPECTIVE FROM LOCKE'S RANGE-OF-AFFECT HYPOTHESIS
}

(Accepted 4 December 2005)

\begin{abstract}
Importance weighting is a common idea in quality of life (QOL) measurement. Based on the common idea that important domains should have more contributions to individuals' QOL total score, the weighting procedure of multiplying item satisfaction by item importance was adopted in many QOL instruments. However, in Locke's [1969, Organizational Behavior and Human Performance 4, pp. 309-336; 1976, in: M.D. Dunnette (eds.), Handbook of Industrial and Organizational Psychology (Rand McNally, Chicago), pp. 1297-1343] range-ofaffect hypothesis, he indicated that the satisfaction evaluation of an item was determined by the have-want discrepancy, importance and their interaction (discrepancy $\times$ importance), implying that item satisfaction has incorporated the judgment of item importance, therefore, weighting an item satisfaction score with an item importance score is unnecessary. The purpose of this study was to examine the range-of-affect hypothesis in the context of QOL research. Three hundred and thirty two undergraduate students at National Taiwan University (NTU) participated in the study. Item satisfaction, importance and perceived have-want discrepancy were measured for 12 different life-area items. Global life satisfaction was measured as well. Regression analysis results showed that item importance and perceived have-want discrepancy have a significant interaction effect on item satisfaction, supporting Locke's range-of-affect hypothesis. In addition, regression analysis results also showed that item importance and item satisfaction did not have a significant interaction effect on global satisfaction, suggesting that weighting item satisfaction score by item importance value does not have advantages in predicting global satisfaction. In a summary, the findings revealed that item satisfaction has incorporated the judgment of item importance, and, thus, the procedure of importance weighting on item satisfaction is unnecessary.
\end{abstract}

KEY WORDS: importance, quality of life, satisfaction, weighting

\section{INTRODUCTION}

'Importance weighting' is a common idea in Quality of life (QOL) research. The basic idea is that items contained in a measurement have different importance for individuals; therefore, in capturing individuals' perception, feeling or evaluation on these items, information on items' importance should be incorporated in the scoring procedure and reflected in the final 
score. Accordingly, 'Importance weighting' was proposed to serve this purpose, and the common procedure is to weight satisfaction score by importance score at item level. Several instruments have adopted this weighting method, such as the Comprehensive Quality Of Life Scale (ComQol, Cummins, 1997), the Quality of life Index (QOL Index, Ferrans and Powers, 1985), the Quality of Life Inventory (QOL Inventory, Frisch, 1992), the Quality of Life Profile-Adolescent version (QOLPAV, Raphael et al., 1996), and the Flanagan Quality of Life Scale (QOLS, reviewed in Dijkers, 2003), to name a few.

In these QOL instruments, QOL is measured as life satisfaction, and in addition to the section of satisfaction rating, they also contain a section of importance rating, in which participants are asked to rate the importance of each item. The weighted score computed by multiplying satisfaction score with importance score for each item is regarded as the individualized QOL score. For example, Frisch et al. (1992) indicated that “The inventory's (the Quality of Life Inventory) scoring scheme reflects the assumption that a person's overall life satisfaction is a composite of the satisfactions in particular areas of life weighted by their relative importance to the individual. Thus, the product of the satisfaction and importance ratings for each area of life is computed."

At first glance, weighting satisfaction score with item importance appears straightforward, so that many instruments adopt this weighting procedure in their scoring system without examining the necessity and appropriateness of this weighting procedure. Recently, $\mathrm{Wu}$ and Yao (in press, a) adopted four commonly used weighting algorithms developed by Cummins (1997), Raphael (1996), Ferrans and Powers (1985) and Frisch (1992) to see if importance-weighted satisfaction score is better than unweighted score for predicting global life satisfaction. The correlation results revealed that the weighted scores computed from different algorithms didn't have higher correlations to global life satisfaction than the unweighted scores. In addition, regression analysis results also revealed that item importance did not have a moderating effect on the relationship between item satisfaction and global life satisfaction, suggesting that item importance has nothing to do with the relationship between item satisfaction and global satisfaction. Their empirical analysis revealed that weighting satisfaction score by importance rating did not have a beneficial effect in predicting global satisfaction.

Moreover, Trauer and Mackinnon (2001) proposed an important characteristic of the relation between item satisfaction and item importance ratings. They showed that extreme dissatisfaction/satisfaction rating was associated with higher importance rating, suggesting that satisfaction rating has incorporated the judgment of importance. In other words, people would 
have strong dissatisfaction/satisfaction evaluation on more important items than the evaluation on less important items. This finding may suggest that satisfaction rating has incorporated the judgment of importance. And accordingly, it may also imply that weighting satisfaction score with item importance is unnecessary. Therefore, if it is the case that satisfaction rating has incorporated the judgment of importance, then, the basis of importance weighting on satisfaction score is not tenable.

In fact, in Locke's $(1969,1976)$ job satisfaction theory, he indicated that satisfaction rating does incorporate the judgment of importance. He proposed the range-of-affect hypothesis to state that responses of an affective evaluation (e.g., satisfaction) reflect a dual value judgment: (1) the discrepancy between what the individual wants and what he/she perceive himself/herself as getting, and (2) the importance of what is wanted to the individual, and also proposed that the level of satisfaction was influenced by the interaction of have-want discrepancy and importance. Specifically, Locke $(1969,1976)$ claimed that, given the amount of discrepancy, items with high personal importance could produce a wide affective reaction ranging from great satisfaction to great dissatisfaction. Items with low personal importance, on the other hand, only produce a restricted affective reaction to the neutral point of the satisfaction-dissatisfaction dimension. In other words, given the amount of discrepancy, range of satisfaction rating on an item is determined by the item importance. Thus, from this perspective, the level of satisfaction for an item is determined not only by the have-want discrepancy, but also by the importance of that item.

The range-of-affect hypothesis has been supported by empirical studies on job satisfaction, in which the results showed that when a direct cognitive comparison between have and want is made, item importance has a moderating effect on the range of satisfaction rating of that item (McFarlin et al., 1995; McFarlin and Rice, 1992; Mobley and Locke, 1970; Rice et al., 1991; Rice et al., 1991). In the seminal work of Mobley and Locke (1970), five studies were designed to examine the range-of-affect hypothesis. The results of the first four studies consistently revealed that the association strength between have-want discrepancy and satisfaction was stronger on higher important items, scenario and situation (in some study, scenarios and situations were used to manipulate the level of importance) than those with less importance. In addition, the result of the fifth study also showed that the variability of satisfaction rating produced by an item would be proportional to the importance of that item, supporting that items with higher importance would have a wider range on dissatisfaction-satisfaction scale. 
In Rice et al. (1991b) study, the results of two $2 \times 2$ (item importance $\times$ amount of discrepancy) factorial experiments also revealed that in the high importance conditions, the discrepancy amount has a stronger relation with satisfaction than in the low importance conditions. In addition, Rice et al. (1991) used regression analyses to test Locke's rangeof-affect hypothesis $(1969,1976)$ by regressing item satisfaction on item discrepancy, item importance and their interaction (item discrepancy $\times$ item importance). Their result showed that the relationship between the amount of item discrepancy and item satisfaction was generally stronger among people who regard the item as higher importance than people who regard the item as lower importance. Further, since item satisfaction has incorporated the judgment of item importance, it is suggested that weighting item satisfaction with item importance is redundant and is no advantage in predicting global satisfaction, Rice et al. (1991) also conducted regression analyses to show that item importance does not influence the degree to which item satisfaction contributes to overall job satisfaction. Therefore, according to the Locke's framework, there are three rules that can be summarized as (1) perceived have-want discrepancy of an item is a significant predictor for item satisfaction, (2) item importance moderates the relationship between perceived have-want discrepancy and item satisfaction, but (3) item importance does not moderate the relationship between item satisfaction and global satisfaction. These three rules were also supported by McFarlin and Rice (1992) and McFarlin et al.'s (1995) studies.

This finding not only exists in the job satisfaction research, but exists in QOL research as well. In Welham et al.'s (2001) study, they asked participants to rate their real status and their ideal status on the same scale, in which four statements were ranged from best (4) to worse (1) condition. For example, regarding social domain's life quality, the four statements were (1) A number of close friends who often keep in touch; (2) One or two close friends who keep in touch; (3) A few not so close friends would be best; (4) No friends would be fine by me. Participants were asked to choose the ideal status and the real status among the four statements. And the gap between the two choices was the discrepancy score for that item. In addition, participants were also asked to rate the importance and satisfaction of that item. Then the correlations between real statue and item satisfaction of each item were compared with those between importance-weighted discrepancy and item satisfaction of each item. The result revealed that importance-weighted discrepancies were more strongly related to item satisfaction than were the real status only, suggesting that weighting have-want discrepancy score with item importance could improve the prediction for item satisfaction. 
This finding was also consistent with Locke's hypothesis that both item importance and item discrepancy determine the level of item satisfaction.

Therefore, in the literature, many empirical studies have supported Locke's theory $(1969,1976)$ on range-of-affect hypothesis, revealing that have-want discrepancy and item satisfaction have stronger association on high-importance items than low-importance items. All these findings indicated that item satisfaction has incorporated the judgment of item importance and implied that weighting item satisfaction score with item importance score is redundant. This may explain why many empirical studies reported that importance-weighted satisfaction score was not superior to unweighted satisfaction score in predicting criterion variables, such as employees' turnover (e.g., Mikes and Hulin, 1968; Waters and Roach, 1971), global job satisfaction (e.g., Ewen, 1967; Waters, 1969; Waters and Roach, 1971; Rice et al., 1991; Staples and Higgins, 1998), and global life satisfaction (Hsieh, 2003; Wu and Yao, in press, a).

However, studies on Locke's range-of-affect hypothesis $(1969,1976)$ were conducted in job satisfaction research. The theoretical relationships among perceived have-want discrepancy, item importance and item satisfaction that postulated in Locke's hypothesis were not directly examined in the QOL research. Consequently, the purpose of this study was to examine Locke's range-of-affect hypothesis directly, to see if the level of item satisfaction is determined by the amount of have-want discrepancy and item importance.

In this study, three hypotheses mentioned earlier were examined, including (1) perceived have-want discrepancy of an item is a significant predictor for item satisfaction, (2) item importance moderates the relationship between perceived have-want discrepancy and item satisfaction, and (3) item importance does not moderate the relationship between item satisfaction and global satisfaction. If the three hypotheses were all supported, it could be concluded that item satisfaction has incorporated the judgment of item importance, and weighting item satisfaction with item importance is unnecessary.

\section{METHOD}

\subsection{Participants and Procedure}

Three hundred and thirty two undergraduate students $(63.3 \%$ female $(n=210)$ and $35.8 \%$ male $(n=119), 3$ participants did not report their gender) at National Taiwan University participated in the study voluntarily. The mean age was 19.80 years $(\mathrm{std}=1.98)$. All questionnaires were filled out during approximately $30 \mathrm{~min}$ and then handed in to the researchers. 


\subsection{Instruments}

2.2.1. Quality of life questionnaire. Since there is no QOL instrument designed for measuring items' have-want discrepancy, importance and satisfaction for the current purpose, a quality of life questionnaire was constructed by referencing facets form World Health Organization Quality of Life questionnaire (WHOQOL-100). Twelve facets were selected. The 12 life facets include (1) energy and fatigue, (2) sleep and rest, (3) work capacity (with learning), (4) social support, (5) physical safety and security, (6) financial resources, (7) health and social care (accessibility and quality), (8) opportunities for acquiring new information and skills, (9) physical environment, (10) home environment, (11) transport, and (12) participation in and opportunities for recreation/leisure. In the first section, participants were asked to rate their satisfaction with these 12 facets (one item for each facet) on a seven-point Likert-type scale ranging from 1 (very dissatisfied) to 7 (very satisfied). In order to capture enough variance on satisfaction, sevenpoint Likert-type scale was used. Further, in the second section, 12 items expressed in the form of direct have-want discrepancy comparison were used to measure the amount of have-want discrepancy for each facet. For example, the item for Energy is "What is the difference between your energy status and the status you want yourself to be?" Participants were asked to rate the 12 direct have-want discrepancy items on a five-point Likert-type scale ranging from -4 (large discrepancy from the wanted status) to 0 (the same with wanted status). In following analysis, the scores were recorded from 5 to 1 to make higher value indicate larger discrepancy. Finally, participants were also asked to rate the importance of each facet on a five-point Likert-type scale ranging from 1 (not at all important) to 5 (very important).

\subsubsection{Satisfaction with Life Scale. The Satisfaction with Life Scale} (SWLS), developed by Diener et al. (1985), is a widely used measure of subjective well being. Based on Shin and Johnson's (1978) perspective, Diener et al. (1985) defined life satisfaction as conscious cognitive judgment of life in which individuals compare their life circumstances with a self-imposed standard. Because different people may have very different ideas about what constitutes a good life, SWLS was developed to assess satisfaction with the individual's life as a whole, creating a global evaluation of life satisfaction (Pavot and Diener, 1993). The scale contained 5 items and employed a 7-point Likert scale with higher values corresponding to a higher degree of satisfaction. The total score was calculated to represent the level of satisfaction, ranging from 5 to 35 . The SWLS has 
shown good reliability and validity. The internal reliability was ranged from 0.79 to 0.89 . The test-retest reliability coefficients of SWLS were 0.83 for 2-week interval, 0.84 for 1-month interval, 0.64 and 0.82 for 2-month interval in different studies (see Pavot and Diener, 1993). Besides, it has shown that SWLS has only one factor and this single-factor solution has been replicated in different studies (Diener et al., 1985; Arrindell et al., 1991; Pavot et al., 1991; Shevlin and Bunting, 1994; Lewis et al., 1995; Shevlin et al., 1998; Arrindell et al., 1999; Atienza et al., 2003; Westaway et al., 2003). Regarding validity, the SWLS demonstrated adequate construct validity, convergent validity and discriminant validity (see Pavot and Diener, 1993; Lucas et al., 1996; Arrindell et al., 1999;). In Hong Kongpublished Chinese translated version, Sachs (2003) has shown there was a single factor underlying the scale and demonstrated good validity of the scale. In addition, $\mathrm{Wu}$ and Yao's study (in press, b) also confirmed the single-factor structure of the SWLS-Taiwan version, and revealed the SWLS-Taiwan version was factor invariant across gender. In this study, the internal consistency (coefficient $\alpha$ ) of the scale was 0.89 .

\section{RESULTS}

\subsection{Descriptive Statistics}

Table I presented the mean and standard deviation of item satisfaction, item discrepancy and item importance. Regarding the SWLS, the mean was 4.19, and standard deviation was 1.21 .

In Table II, item importance was correlated with item satisfaction and item discrepancy for 12 items, respectively. According to the result, item importance generally has low or non-significant relations with item satisfaction and item discrepancy, which was consistent with the findings of McFarlin et al. (1995). In Table III, item satisfaction was correlated with item discrepancy for 12 items. According to the result, item satisfaction had strong correlations with item discrepancy.

\subsection{Have-Want Discrepancy and Item Satisfaction}

In this section, the first hypothesis: that "perceived have-want discrepancy of an item is a significant predictor for item satisfaction" was examined. In fact, this hypothesis was supported by the result of the previous section. Table III shows that for each item, item satisfaction was strongly correlated with item discrepancy (correlations ranged from -0.73 to $-0.51, p<0.01$ ). 


\section{TABLE I}

Descriptive Statistics of Item Satisfaction, Discrepancy, and Importance Ratings

\begin{tabular}{|c|c|c|c|c|c|c|}
\hline \multirow[t]{2}{*}{ Items } & \multicolumn{2}{|c|}{ Satisfaction } & \multicolumn{2}{|c|}{ Discrepancy } & \multicolumn{2}{|c|}{ Importance } \\
\hline & Mean & Std. & Mean & Std. & Mean & Std. \\
\hline 1. Energy & 4.25 & 1.38 & 3.00 & 1.04 & 4.09 & 0.77 \\
\hline 2. Sleep & 3.89 & 1.54 & 3.01 & 1.22 & 4.27 & 0.80 \\
\hline 3. Learning & 4.23 & 1.33 & 2.81 & 1.14 & 4.41 & 0.66 \\
\hline 4. Social support & 5.05 & 1.39 & 2.03 & 1.12 & 3.93 & 1.06 \\
\hline 5. Safety and security & 4.92 & 1.21 & 2.26 & 1.00 & 3.83 & 0.90 \\
\hline 6. Financial resources & 4.38 & 1.49 & 2.43 & 1.19 & 4.02 & 0.82 \\
\hline 7. Health care & 4.83 & 1.22 & 1.93 & 0.90 & 3.62 & 0.92 \\
\hline 8. Information & 5.01 & 1.23 & 1.86 & 0.92 & 3.92 & 0.88 \\
\hline 9. Physical environment & 3.48 & 1.33 & 3.22 & 1.13 & 3.85 & 0.93 \\
\hline 10. Living condition & 4.31 & 1.44 & 2.68 & 1.11 & 4.00 & 0.83 \\
\hline 11. Transport & 4.47 & 1.38 & 2.30 & 1.03 & 3.89 & 0.84 \\
\hline 12. Recreation & 4.29 & 1.35 & 2.53 & 1.07 & 4.09 & 0.86 \\
\hline
\end{tabular}

\subsection{Item Importance, Have-Want Discrepancy and Item Satisfaction}

In this section, the second hypothesis that "item importance moderates the relationship between perceived have-want discrepancy and item satisfaction" was examined by regression analysis, in which item satisfaction was regressed on item discrepancy, item importance and their interaction (item

\section{TABLE II}

Correlations between Item Importance and Item Satisfaction and Item Discrepancy

\begin{tabular}{lcc}
\hline Item (importance rating) & Item satisfaction & Item discrepancy \\
\hline 1. Energy & $0.14^{* *}$ & -0.02 \\
2. Sleep & 0.03 & 0.08 \\
3. Learning & 0.00 & $0.11^{*}$ \\
4. Social support & $0.50^{* *}$ & $-0.28^{* *}$ \\
5. Safety and security & 0.00 & $0.11^{*}$ \\
6. Financial resources & $-0.19^{* *}$ & $0.29^{* *}$ \\
7. Health care & $0.16^{* *}$ & -0.04 \\
8. Information & $0.16^{* *}$ & 0.02 \\
9. Physical environment & -0.10 & $0.23^{* *}$ \\
10. Living condition & -0.06 & 0.03 \\
11. Transport & 0.03 & 0.07 \\
12. Recreation & $0.14^{* *}$ & 0.03 \\
\hline
\end{tabular}

Note: ${ }^{*} p<0.05 ; * * p<0.01$. 
TABLE III

Correlations between Item Satisfaction and Item Discrepancy

\begin{tabular}{ll}
\hline Item (satisfaction rating) & Item discrepancy \\
\hline 1. Energy & $-0.56^{* *}$ \\
2. Sleep & $-0.70^{* *}$ \\
3. Learning & $-0.64^{* *}$ \\
4. Social support & $-0.73^{* *}$ \\
5. Safety and security & $-0.45^{* *}$ \\
6. Financial resources & $-0.72^{* *}$ \\
7. Health care & $-0.51^{* *}$ \\
8. Information & $-0.60^{* *}$ \\
9. Physical environment & $-0.62^{* *}$ \\
10. Living condition & $-0.66^{* *}$ \\
11. Transport & $-0.65^{* *}$ \\
12. Recreation & $-0.59^{* *}$ \\
\hline
\end{tabular}

Note: All correlation coefficients were significant at ${ }^{* *} p<0.01$.

discrepancy $\times$ item importance). In order to avoid the collinearity resulting from the interaction term, raw scores of item discrepancy and item importance were centered in each model by subtracting the mean value from item discrepancy and item importance scores, and the interaction term (item discrepancy $\times$ item importance) was computed from the centered scores.

The result of regression analysis was presented in Table IV. It showed that item importance had significant moderation effects on eight items,

TABLE IV

Coefficient of Moderated Regression Models of Item Satisfaction

\begin{tabular}{llllll}
\hline Items & Constant & Discrepancy & Importance & Dis $\times$ Imp & $R^{2}$ \\
\hline 1. Energy & $4.25^{* *}$ & $-0.72^{* *}$ & $0.23^{* *}$ & $-0.16^{*}$ & 0.34 \\
2. Sleep & $3.89^{* *}$ & $-0.88^{* *}$ & $0.15^{* *}$ & -0.11 & 0.51 \\
3. Learning & $4.23^{* *}$ & $-0.76^{* *}$ & 0.15 & 0.01 & 0.41 \\
4. Social support & $5.01^{* *}$ & $-0.82^{* *}$ & $0.43^{* *}$ & $-0.09^{* *}$ & 0.63 \\
5. Safety and security & $4.94^{* *}$ & $-0.53^{* *}$ & 0.04 & $-0.17^{*}$ & 0.22 \\
6. Financial resources & $4.43^{* *}$ & $-0.86^{* *}$ & -0.02 & $-0.19^{* *}$ & 0.53 \\
7. Health care & $4.82^{* *}$ & $-0.68^{* *}$ & $0.17^{* *}$ & -0.12 & 0.28 \\
8. Information & $5.01^{* *}$ & $-0.79^{* *}$ & $0.24^{* *}$ & $-0.15^{*}$ & 0.40 \\
9. Physical environment & $3.51^{* *}$ & $-0.72^{* *}$ & 0.07 & $-0.11^{*}$ & 0.39 \\
10. Living condition & $4.31^{* *}$ & $-0.83^{* *}$ & -0.05 & $-0.18^{* *}$ & 0.45 \\
11. Transport & $4.48^{* *}$ & $-0.85^{* *}$ & 0.10 & -0.12 & 0.43 \\
12. Recreation & $4.28^{* *}$ & $-0.72^{* *}$ & $0.22^{* *}$ & $-0.24^{* *}$ & 0.39 \\
\hline
\end{tabular}

${ }^{*} p<0.05 ; * * p<0.01$. 
including Energy, Social support, Safety and security, Financial resources, Information, Physical environment, Living condition and Recreation. From the regression analysis results of these items, it can be inferred that the negative relation between item discrepancy and item satisfaction was stronger when item importance became higher, which was consistent with Locke's hypothesis that item importance moderates the relationships between item discrepancy and item satisfaction.

\subsection{Item Importance, Item Satisfaction and Global Satisfaction}

The purpose of this section was to examine the hypothesis that 'item importance does not moderate the relationship between item satisfaction and global satisfaction' by conducting regression analysis. In the beginning, correlation analysis was conducted to understand the relationship between item satisfaction and the SWLS (global satisfaction) and the relationship between item importance and the SWLS. The result was presented in Table V, in which item satisfaction was significantly correlated with the SWLS (correlations ranged from 0.25 to 0.45 ), and item importance was non-significantly or lowly correlated with the SWLS (correlations ranged from -0.03 to 0.23 ).

Further, in regression analysis, the score of the SWLS was regressed on item satisfaction, item importance and the interaction term (item satisfaction $\times$ item importance) for each item. Similarly, centered scores of item satisfaction and item importance were used in regression models. The result

TABLE V

Correlations between Item Satisfaction/Importance and the SWLS

\begin{tabular}{llc}
\hline Items & Item satisfaction & Item importance \\
\hline 1. Energy & $0.37^{* *}$ & $0.13^{*}$ \\
2. Sleep & $0.28^{* *}$ & 0.06 \\
3. Learning & $0.38^{* *}$ & 0.09 \\
4. Social support & $0.34^{* *}$ & $0.23^{* *}$ \\
5. Safety and security & $0.25^{* *}$ & $0.21^{* *}$ \\
6. Financial resources & $0.37^{* *}$ & -0.03 \\
7. Health care & $0.29^{* *}$ & $0.13^{*}$ \\
8. Information & $0.30^{* *}$ & 0.09 \\
9. Physical environment & $0.28^{* *}$ & 0.10 \\
10. Living condition & $0.29^{* *}$ & 0.09 \\
11. Transport & $0.30^{* *}$ & 0.07 \\
12. Recreation & $0.45^{* *}$ & 0.06 \\
\hline
\end{tabular}

Note: ${ }^{*} p<0.05 ; * * p<0.01$. 
is presented in Table VI, in which only one item had significant interaction effect, showing that item importance, generally, did not moderate the relationship between item satisfaction and global satisfaction.

\section{DISCUSSION}

\subsection{The Role of Importance Weighting and Importance Rating}

The purpose of this study was to examine three hypotheses derived from Locke's theory $(1969,1976)$. According to the current results, it could be concluded that the three hypotheses (1) perceived have-want discrepancy of an item is a significant predictor for item satisfaction; (2) item importance moderates the relationship between perceived have-want discrepancy and item satisfaction, and (3) item importance does not moderate the relationship between item satisfaction and global satisfaction, were generally supported. These findings showed that degree of importance exacerbates the contribution of item discrepancy on item satisfaction. In other words, given the amount of item discrepancy, people who regard the item as higher importance would have stronger satisfaction/dissatisfaction than people who regard the item as lower importance, implying that item satisfaction has already reflected the influence of item have-want discrepancy and item importance. Therefore, importance weighting on item satisfaction is unnecessary.

TABLE VI

Coefficient of Moderated Regression Models of the SWLS

\begin{tabular}{llllll}
\hline Items & Constant & Item_sat & Item_imp & Item_sat $\times$ Item_imp & $R^{2}$ \\
\hline 1. Energy & $4.20^{* *}$ & $0.33^{* *}$ & 0.11 & -0.05 & 0.15 \\
2. Sleep & $4.19^{* *}$ & $0.22^{* *}$ & 0.08 & 0.00 & 0.08 \\
3. Learning & $4.19^{* *}$ & $0.34^{* *}$ & 0.17 & 0.06 & 0.16 \\
4. Social support & $4.19^{* *}$ & $0.27^{* *}$ & 0.08 & 0.00 & 0.12 \\
5. Safety and security & $4.19^{* *}$ & $0.25^{* *}$ & $0.29^{* *}$ & -0.03 & 0.10 \\
6. Financial resources & $4.19^{* *}$ & $0.31^{* *}$ & 0.07 & -0.02 & 0.14 \\
7. Health care & $4.19^{* *}$ & $0.27^{* *}$ & 0.11 & 0.02 & 0.09 \\
8. Information & $4.19^{* *}$ & $0.29^{* *}$ & 0.06 & 0.00 & 0.09 \\
9. Physical environment & $3.96^{* *}$ & $0.25^{* *}$ & 0.06 & $0.11^{*}$ & 0.10 \\
10. Living condition & $4.19^{* *}$ & $0.25^{* *}$ & $0.15^{*}$ & -0.02 & 0.10 \\
11. Transport & $4.19^{* *}$ & $0.26^{* *}$ & 0.08 & 0.02 & 0.09 \\
12. Recreation & $4.18^{* *}$ & $0.39^{* *}$ & 0.01 & 0.08 & 0.21 \\
\hline
\end{tabular}

${ }^{*} p<0.05 ; * * p<0.01$. 
In addition to range-of-affect hypothesis, Trauer and Mackinnon (2001) also suggested that weighting satisfaction score by importance rating is undesirable and unnecessary from a statistical perspective. They proposed that the weighted score computed by multiplying satisfaction score and importance score do not have the ratio property, because the scales of satisfaction rating and importance rating are interval scale. Thus, it is difficult to interpret the weighted score without a clear scaling property. Moreover, multiplying satisfaction and importance scores could mask the fact that people with the same weighted score could have quite different experiences of QOL (Streiner and Norman, 1995). For example, the weighted score of a person who have 2 point on satisfaction rating and 5 point on important rating is the same as the weighted score of the other person who have 5 point on satisfaction rating and 2 point on important rating. In fact, in considering the drawback of interpretation, the WHOQOL group did not use importance-weighted score as the final QOL score in the WHOQOL (Skevington et al., 2004), although it contains a section for importance rating. Moreover, Trauer and Mackinnon (2001) also demonstrated that correlations of the weighted scores with third variable vary systematically with the magnitudes of the means and SDs of importance rating, implying that changing the zero-point on the interval scale of importance rating distorts the relationships between the weighted score and other variables. This phenomena was also mentioned and expressed by statistical formula in Wu and Yao's study (in press, a). Therefore, according to the reasons mentioned above, weighting satisfaction score by importance rating is not appropriate.

However, we did not imply that importance rating is not important in assessing individuals' QOL. In fact, information of item importance is valuable for understanding an individual's QOL. According to Locke's range-of-affect hypothesis, satisfaction with a life area (an item) was determined by the degree of have-want discrepancy and the importance of that area, suggesting that the item discrepancy and item importance were the two components that result in the observed degree of satisfaction. Accordingly, it could be said that a satisfaction-based QOL score is not sufficient to understand an individual's perception on that life area, since a particular satisfaction level could result from a small have-want discrepancy with higher importance or a large have-want discrepancy with lower importance. Therefore, acquiring the information of item discrepancy and item importance could lead researchers to evaluate and understand an individual's QOL with an informative background. 
In addition, Skevington et al. (2004) indicated that information about item importance is useful for instrument developers or researchers to evaluate if items are relevant to the research sample and population for whom the scale is intended. Moreover, item importance information is also useful to identify the value differences among different diseased, social or cultural groups. For example, Saxena et al. (on behalf of the WHOQOL Group, 2001) investigated the difference of values on life domains among age, gender and center (cultural) groups by the importance rating of WHOQOL-BREF (World Health Organization Quality of Life questionnaire - brief version) items.

Further, information about item importance would also assist health services providers to design appropriate health services for an individual or a group, and then, enhance the efficiency of health care. Oishi et al. (1999) had indicated that intra-individual changes in satisfaction were strongly influenced by the degree of success in the domains that individual's value. This implies that understanding which life area is more important and enhancing the performance of this area are crucial for QOL promotion. In a summary, in arguing that weighting item satisfaction with item importance is unnecessary, it does not imply that the information of item importance is not valuable for QOL assessment.

\subsection{Limitations}

There are several limitations that should be mentioned. First, the members of the current sample were NTU students. Although Locke's hypothesis was supported in this sample, this homogenous sample may restrict the moderating effect of item importance. This is because life experiences of students will be more similar than other populations and the similarity of experience will not provide enough variance to detect the theoretical effect. Therefore, a heterogeneous sample is desired to examine Locke's range-of-affect hypothesis in the future.

Second, the measurement of item importance in this study was based on rating method. In Rice, Gentile, and McFarlin's (1991) study they used seven alternative measures to assess item importance, including four rating methods, two ranking methods, and a point-distribution method. Although they recommend the direct rating method (as the importance rating in the current study) for measuring item importance when respondent time and simplicity of administration were concerned, however, they also indicated that the composite importance value (computed from the seven measures) has better performance than any individual measure. Thus, in future studies, different measures of item importance can be used to compute a 
higher-reliability composite value. This composite measure may lead to a better performance in detecting the moderating effect of item importance.

Third, although the findings of this study generally supported Locke's hypotheses, so far, all the findings were found in the inter-personal (between-subject) framework. Locke's range-of-affect hypothesis (1969, 1976) could be expressed in the intra-personal (within-subject) framework as well. For QOL research, the intra-personal framework can be more meaningful. The reason is that, in QOL research, an individual's QOL score is usually computed from various life areas. If Locke's hypothesis is supported in the intra-personal framework, it could be suggested that weighting item satisfaction score with item importance is unnecessary for an individual. Therefore, in the future study, within-subject experiments should be designed to test Locke's hypothesis.

Finally, in the quality of life research, the Multiple Discrepancies Theory (MDT; Michalos, 1985) also mentioned the importance of discrepancies on satisfaction. However, it emphasized different forms of discrepancies from different aspects, such as discrepancy between self and others, discrepancy between present and past, and so on, and explained how these discrepancies directly and indirectly influence satisfaction. In brief, the MDT stressed the mediating role of the discrepancies on the satisfaction. However, the important weighting we addressed here is the "moderating role of importance" on the "mediation effect of the discrepancies on the satisfaction". Although Michalos (1985) also mentioned the importance weighting in his research, however, the weighting value of each component he used was assigned by researcher, not by respondents' judgments. In addition, the weighting values were gave on the different forms of discrepancies, not on the life domains we used in the current study. Thus, we did not focus our attention on the MDT for discussing the role of importance weighting in the context we discussed. However, in the future study, it is worth incorporating the different forms of discrepancies proposed by the MDT to address the issue of important weighting.

\subsection{Implications}

From the angle of Locke's range-of-affect hypothesis, it worth discussing QOL promotion. According to Locke's hypothesis, there are two components that influence the degree of satisfaction on a specific life area. The first one is have-want discrepancy; the other one is the value (importance) of that area. Accordingly, we can promote an individual QOL by "have-want discrepancy" pathway and "value perception" pathway. 
On the "have-want discrepancy" pathway, the gap between present condition and wanted condition affects an individual's satisfaction. It suggests that promoting the present condition or demoting the wanted standard could lead to QOL enhancement. In promoting the present condition, we can help individuals attain the wanted status for making their present circumstance to match their ideal condition. In demoting the wanted standard, we can help individuals set appropriate goals. This is a straightforward approach to enhance an individual's QOL. On the "value perception" pathway, we can enhance an individual's QOL by shifting their importance perception on life areas. This approach does not change their original wanted standards of each area, but adjusts the importance hierarchy of life areas. By this approach, an individual can experience higher QOL without changing their goals of each life area.

However, these two pathways are not wholly independent. From the perspective of self-regulation theory, these two pathways can be incorporated into the process of goal disengagement and reengagement. In this perspective, the goal can be regarded as the wanted status of life and the change of QOL can be considered as a process of goal attainment. Accordingly, if the goal is unattainable, then, an individual's QOL would be worse. However, the two strategies of "goal disengagement" and "goal reengagement" would be helpful in facing this situation. Goal disengagement can be regarded as abandoning the original wanted status of life areas or shifting their attention to others life areas with small gap between realidea statuses. And goal reengagement can be regarded as pursuing the new goal with high involvement. Wrosch et al. (2003) indicated that when confronting an unattainable goal, disengagement from pursing it may help a person (1) avoid accumulate failure experience, (2) redefine the goal as not necessary for satisfaction in life, (3) accommodate to the inability of reaching the goal, and (4) reallocate resources that can be used to promote beneficial effect in other areas of life. Many studies have reported beneficial effect of disengagement from unattainable goals (see Wrosch et al., 2003). However, not only the "goal disengagement", but also the "goal reengagement" plays an important role on QOL promotion. Wrosch et al.'s (2003) cited Carver and Scheier's argument that "Other things being equal, the occurrence of an unattainable goal may be particularly problematic if a person has no valuable alternative goals available toward which thoughts and energy can be redirected. If a self-relevant goal proves unattainable and a person is not able to engage in other meaningful activities, then the person may experience high levels of psychological distress." Therefore, although abandoning the unattainable goals is helpful for avoiding negative consequences, 
however, reengaging a meaning goal is important as well. In Wrosch et al.'s (2003) study, they found that both goal disengagement and goal reengagement were associated with ratings of high subjective well-being. Thus, on QOL promotion, it is helpful for an individual to change their goal in enhancing their QOL.

In summary, the current study examined Locke's range-of-affect hypothesis on the issue of importance weighting. According to the results and above discussion, it is obvious that what Locke $(1969,1976)$ proposed in the range-of-affect hypothesis is a psychological mechanism for satisfaction evaluation, and, according to this mechanism we also can facilitate individuals' QOL by the way of regulating goal attainment.

\section{ACKNOWLEDGEMENTS}

We thank Li-jen Weng, Luo Lu, Chien-chih Kuo, and Chung-ping Cheng for their comments on the original version of the Master's Thesis. We also thank for Kevin Lax's help in editing the English. This study was supported by National Science Council (NSC 94-2413-H-002-018) and the National Health Research Institute (NHRI-EX94-9204PP).

\section{REFERENCES}

Arrindell, W.A., J. Heesink and J.A Feij: 1999, 'The Satisfaction With Life Scale (SWLS): Appraisal with 1700 health young adults in the Netherlands', Personality and Individual Differences 26, 815-826.

Arrindell, W.A., L. Meeuwesen and F.J. Huyse: 1991, 'The Satisfaction with Life Scale (SWLS): Psychometric properties in a non-psychiatric medical outpatients sample', Personality and Individual Differences 12, 117-123.

Atienza, F.L., I. Balaguer and M.L. Garcia-Merita: 2003, 'Satisfaction with Life Scale: analysis of factorial invariance across sexes', Personality and Individual Differences 35, 1255-1260.

Cummins, R.A.: 1997, Comprehensive Quality of Life Scale - Adult: Manual (University Australia, Deakin).

Diener, E., R.A. Emmons, R.J. Larsen and S. Griffin: 1985, 'The Satisfaction with Life Scale', Journal of Personality Assessment 49, 71-75.

Dijkers, M.P.: 2003, 'Individualization in quality of life measurement: Instruments and approaches', Archives of Physical Medicine and Rehabilitation 84, S3-14.

Ewen, R.B.: 1967, 'Weighting components of job satisfaction', Journal of Applied Psychology $51,68-73$.

Ferrans, C. and M. Powers: 1985, 'Quality of Life Index: Development and psychometric properties', Advances in Nursing Science 8, 15-24.

Frisch, M.B.: 1992, 'Use of the Quality of Life Inventory in problem assessment and treatment planning for cognitive therapy of depression', in A. Freeman and F.M. Dattlio (eds.), Comprehensive Casebook of Cognitive Therapy (Plenum Press, New York). 
Frisch, M.B., J. Cornell, M. Villanueva and P.J. Retzlaff: 1992, 'Clinical validation of the Quality of Life Inventory: A measure of life satisfaction for use in treatment planning and outcome assessment', Psychological Assessment 4, 92-101.

Hsieh, C.M.: 2003, 'Counting importance: The case of life satisfaction and relative domain importance', Social Indicators Research 61, 227-240.

Lewis, C.A., M.E. Shevlin, B.P. Bunting and S. Joseph: 1995, 'Confirmatory factor analysis of the satisfaction with life scale: replication and methodological refinement', Perceptual and Motor Skills 80, 304-306.

Locke, E.A.: 1969, 'What is job satisfaction?', Organizational Behavior and Human Performance 4, 309-336.

Locke, E.A.: 1976, 'The nature and causes of job satisfaction', in M.D. Dunnette (ed.), Handbook of Industrial and Organizational Psychology (Rand McNally, Chicago), pp. $1297-1343$.

Lucas, R.E., E. Diener and E. Suh: 1996, 'Discriminant validity of well-being measures', Journal of Personality and Social Psychology 71, 616-628.

McFarlin, D.B., E.A. Coster, R.W. Rice and T. Coopper-Alison: 1995, 'Facet importance and job satisfaction: Another look at the range of affect hypothesis', Basic and Applied Social Psychology 16, 489-502.

McFarlin, D.B. and R.W. Rice: 1992, 'The role of facet importance as a moderator in job satisfaction processes', Journal of Organizational Behavior 13, 41-54.

Michalos, A.C: 1985, 'Multiple discrepancy theory (MDT)', Social Indicators Research 16, 347 413.

Mikes, P.S. and C.L. Hulin: 1968, 'Use of importance as weighting component of job satisfaction', Journal of Applied Psychology 52, 394-398.

Mobley, W.H. and E.A. Locke: 1970, 'The relationship of value importance to satisfaction', Organisational Behavior and Human Performance 5, 463-483.

Oishi, S., E. Diener, E. Suh and R.E. Lucas: 1999, 'Value as a moderator in subjective wellbeing', Journal of Personality 67, 157-184.

Pavot, W. and E. Diener: 1993, 'Review of the satisfaction with life scale', Psychological Assessment 5, 164-172.

Pavot, W., E. Diener, C.R. Colvin and E. Sandvik: 1991, 'Further validation of the Satisfaction evidence for the cross-method convergence of well-being', Social Indicators Research 28, 120.

Raphael, D., E. Rukholm, I. Brown, P. Hill-Bailey and E. Donato: 1996, 'The quality of life profile-Adolescent version: Background, description, and initial validation', Journal of Adolescent Health 19, 366-375.

Rice, R.W., D.A. Gentile and D.B. McFarlin: 1991a, 'Facet importance and job satisfaction', Journal of Applied Psychology 76, 31-39.

Rice, R.W., K. Markus, R.P. Moyer and D.B. McFarlin: 1991b, 'Facet importance and job satisfaction: Two experimental tests of Locke's range of affect hypothesis', Journal of Applied Social Psychology 21, 1977-1987.

Sachs, J: 2003, 'Validation of the Satisfaction with Life Scale in a sample of Hong Kong University students', Psychologia 46, 225-234.

Saxena, S., D. Carlson, R. Billington and J. Orley, on behalf of the WHOQOL Group: 2001, 'The WHO quality of life assessment instrument (WHOQOL-Bref): The importance of its items for cross-cultural research', Quality of Life Research 10, 711-721.

Shevlin, M.E., V. Brunsden and J.N.V Miles: 1998, 'Satisfaction with Life Scale: analysis of factorial invariance, mean structures and reliability', Personality and Individual Differences 25, 911-916.

Shevlin, M.E. and B.P. Bunting: 1994, 'Confirmatory factor analysis of the satisfaction with life scale', Perceptual and Motor Skills 79, 1316-1318. 
Shin, D.C. and D.M. Johnson: 1978, 'Avowed happiness as an overall assessment of the quality of life', Social Indicators Research 5, 475-492.

Skevington, S.M. and K. O'connell, the WHOQOL Group: 2004, 'Can we identify the poorest quality of life? Assessing the importance of quality of life using the WHOQOL-10', Quality of Life Research 13, 23-34.

Staples, D.S. and C.A. Higgins: 1998, 'A study of the impact of factor importance weightings on job satisfaction measures', Journal of Business and Psychology 13, 211-232.

Streiner, D.L. and G.R. Norman: 1995, Health measurement scales: A practical guide to their development and use, 2nd edition (Oxford University Press, New York).

Trauer, T. and A. Mackinnon: 2001, 'Why are we weighting? The role of importance ratings in quality of life measurement', Quality of life research 10, 579-585.

Waters, L.K.: 1969, 'The utility of importance weights in predicting overall job satisfaction and dissatisfaction', Educational and Psychological Measurement 29, 519-522.

Waters, L.K. and D. Roach: 1971, 'Comparison of unweighted and importance- weighted job satisfaction measures for three samples of female office workers', Psychological Reports 28, 779-782.

Westaway, M.S., C. Maritz and N.J. Golele: 2003, 'Empirical testing of the satisfaction with life scale: A South African pilot study', Psychological Reports 92, 551-554.

Welham, J., M. Haire, D. Mercer and T. Stedman: 2001, 'A gap approach to exploring quality of life in mental health', Quality of Life Research 10, 421-429.

Wrosch, C., M.F. Scheier, G.E. Miller, R. Schulz and C.S Carver: 2003, 'Adaptive selfregulation of unattainable goals: Goal disengagement, goal re-engagement, and subjective well-being', Personality and Social Psychology Bulletin 29, 1494-1508.

Wu, C.H. and G. Yao: in press (a), 'Do we need to weight satisfaction scores with importance ratings in measuring quality of life?', Social Indicators Research.

Wu, C.H. and G. Yao: in press (b), 'Analysis of factorial invariance across gender in the Taiwan version of the Satisfaction with Life Scale', Personality and Individual Differences.

Department of Psychology

G. Yao

National Taiwan University

C.H. $\mathrm{Wu}$

1, Sec. 4, Roosevelt RD

Taipei, Taiwan

E-mail:kaiping@ntu.edu.tw 